\title{
TRIPLE PHASE DYNAMIC COMPUTED TOMOGRAPHIC PERFUSION CHARACTERISTICS OF SPIROCERCOSIS INDUCED ESOPHAGEAL NODULES IN NON-NEOPLASTIC VERSUS NEOPLASTIC CANINE CASES
}

\author{
Robert M. Kirberger ${ }^{*}$, Nicky Cassel, Nerissa Stander, Melanie McLean and Eran Dvir \\ Department of Companion Animal Clinical Studies, Faculty of Veterinary Science, University of Pretoria, \\ Onderstepoort, Republic of South Africa

\begin{abstract}
*Address correspondence and reprint requests to Robert M. Kirberger, at the above address. E-mail: robert.kirberger@up.ac.za
\end{abstract}

Dr. Dvir's current address is The Animal Welfare Unit, Ministry of Agriculture, Veterinary Services and Animal Health, P.O.B 12, Beit Dagan, 50250, Israel.

Dr. Stander's current address is Western Australian Veterinary Emergency and Specialty, Unit 1, 640 Beeliar Drive, 6164, Perth, Western Australia.

Funding sources: The senior author received funding from the South African National Research Foundation for this project.

Keywords: dynamic CT; dog; esophageal nodule; neoplastic transformation; perfusion; Spirocerca lupi

\begin{abstract}
Neoplastic transformation of Spirocerca lupi induced esophageal nodules carries a poor prognosis. Clinical, clinicopathological, endoscopic, and radiographic characteristics may be indicative of neoplastic transformation but variable sensitivity and specificity of these parameters makes their use questionable. We hypothesized that CT would be a better diagnostic modality to discriminate between non-neoplastic and neoplastic nodules. In this prospective study of $38 \mathrm{dogs}$, the appearance and perfusion characteristics of confirmed spirocercosis-induced neoplastic and non-neoplastic esophageal nodules were described using survey CT and triple phase dynamic CT angiography (CTA). Pre- and post-contrast early arterial, late arterial, and venous CTA images were evaluated. Non-neoplastic nodules were smooth and nonmineralized with a higher proportion of hypoattenuating necropurulent cavities compared to neoplastic nodules that had a more irregular surface, with $93 \%$ having mineralized foci and rarely any hypoattenuating pockets. Non-neoplastic nodules were significantly more perfused than neoplastic nodules with the difference being up to 23 Hounsfield units. The difference was most marked in the early and late arterial phases $(P=$ 0.0005 and 0.00005 , respectively). Ratios of the normal esophagus adjacent to the neoplastic and non-neoplastic nodules did not differ significantly from each other. Perfusion findings demonstrated relative hypoperfusion of the esophageal sarcomas. Findings from the current study indicated that CT characteristics of relative postcontrast hypoperfusion, combined with nodule irregularity and mineralization warrant a high level of concern for neoplastic transformation in canine spirocercosis-induced esophageal nodules.
\end{abstract}




\section{Introduction}

Spirocerca lupi (S. lupi) is a nematode with worldwide distribution but is most commonly found in tropical and subtropical areas. Dogs are definitive hosts and become infected by ingesting the intermediate host (coprophagous beetles) or paratenic host (lizards, wild, and domestic birds, etc.).[1,2] After ingestion the larvae migrate from the stomach via the celiac artery to the abdominal aorta from where they migrate intramurally cranially to the caudal thoracic aorta where they remain for up to 3 months whilst maturing to young adults.[1] The young adults then penetrate the adjacent esophagus where they form a fibroblastic nodule or nodules which may progress to an esophageal sarcoma.[3] The diagnosis of the latter, particularly in the earlier stages remains challenging. The diagnosis of $S$. lupi esophageal pathology is confirmed by finding the typical eggs in the feces[5] or by means of gastroesophageal endoscopy. $[2,4]$ In endemic areas aortic aneurysm and caudal thoracic spondylitis on thoracic radiographs together with the typical caudodorsal mediastinal mass confirm the diagnosis.[2, 4, 6] Thoracic CT is a useful modality to evaluate possible metastasis, as well as esophageal and aortic pathology.[2, 7] On endoscopy neoplastic transformation is seen as increased nodule size and cauliflower-like shape with necrotic areas whereas nonmalignant nodules have a smoother appearance.[8] Additionally a variety of clinical parameters and growth factors have been assessed in an attempt to determine accurate markers for neoplastic transformation.[9-11] Although changes in some of the parameters were indicative of malignancy a definite diagnostic tool to distinguish non-neoplastic from neoplastic nodules is still lacking. Benign lesions can be successfully treated with avermectins. $[2,12,13]$ However malignant transformation can only be treated surgically with or without chemotherapy and generally with poorer outcomes.[8] These differences emphasize the need for improved methods to distinguish non-neoplastic from neoplastic nodules ante-mortally to enable early treatment options for malignant cases as well as to get a better understanding of the pathophysiology of neoplastic transformation. In man esophageal cancer is a common malignant neoplasm.[14] Squamous cell carcinomas are most commonly reported and adenocarcinomas also occur.[14] However, the opposite is true in dogs where primary, and to a lesser extent metastatic, esophageal neoplastic lesions are extremely rare in nonendemic spirocerca areas making up less than $0.3 \%$ of reported tumors.[15] In endemic spirocerca areas neoplastic transformation of the spirocerca induced esophageal nodule to a sarcoma may occur in up to $25 \%$ of cases.[16] These are usually osteosarcomas, less commonly fibrosarcoma or undifferentiated sarcoma and even a chondrosarcoma has been reported. $[8,17]$

Evaluation of tumors can be done by assessing tumor perfusion which correlates directly to tumor angiogenesis which in turn is essential for neoplastic growth and metastasis.[18] Potential tumor perfusion can be evaluated by histological microvessel density (MVD) to identify vascular endothelium in tissues.[10] In man blood vessel density is a significant prognostic indicator in a variety of carcinomas, including those affecting the esophagus.[19] However the same is not true for sarcomas. This may be due to the fact that the microvessels are generally more diffusely spread in sarcomas whereas in carcinomas the vessels tend to be clustered.[20,21] We have shown that $S$. lupi associated sarcomas have significantly lower MVD than in preneoplastic nodules.[10] This was in contrast to the elevated vascular endothelial growth factor expression in both tissue and serum. These conflicting results warrant additional functional studies. Noninvasive indirect assessment of tumor angiogenesis by evaluating tissue perfusion can be done using dynamic CT angiography (CTA). The changes in contrast enhancement that result from tumor angiogenesis can be used to provide diagnostic and prognostic information for cancer patients.[18] Historically imaging tissue 
perfusion has been done in two ways. The first is dynamic CTA at a specific location within the mass over time allowing attenuation curves to be calculated at that location.[18] The second is triple phase angiography where the whole mass is scanned at three different perfusion stages. Comparative regions of interest (ROI) are then made of several slices of the mass to assess tissue perfusion as evidenced by an increase in Hounsfield units (HU) during each scan phase. This allows for a more representative sample of tumor tissue perfusion.[14] Except for dynamic pituitary perfusion studies, neoplasm tissue perfusion reports using CTA are rare in the veterinary literature.[22-24] One study looked at dynamic CTA at the largest cross section of a variety of spontaneous canine tumors (carcinomas, soft tissue, and bone sarcomas) over time.[25] Contrast enhancement was assessed during first pass, wash out and late phases and time attenuation curves calculated for the tumor and contralateral artery ROIs. This study showed that time to peak ratio was significantly higher in carcinomas than sarcomas and that perfusion differed significantly between carcinomas and sarcomas with soft tissue sarcomas being less, and bone sarcomas being more perfused than carcinomas. Another study in dogs attempted to distinguish benign and malignant pelvic masses by comparing pre- and post-contrast images to each other, amongst a variety of other criteria. This was not a triple phase study and the angiography component showed greater inhomogeneity of contrast uptake in neoplastic masses.[26] The above simple perfusion techniques are significantly constrained by the effects of interpatient variations in cardiac output and blood volume.[18] However, evaluation of veterinary patients is often constrained by procedural costs and less sophisticated equipment making the above measurements easier to use.

We hypothesize that post contrast CTA early and late arterial and venous perfusion characteristics of spirocerca induced non-neoplastic and neoplastic nodules may be an accurate method of distinguishing non-neoplastic from neoplastic nodules and that neoplastic nodules will be hypoperfused relative to their non-neoplastic counterparts. The objective of this prospective study was to determine if CT and CTA perfusion characteristics could distinguish between spirocerca induced non-neoplastic and neoplastic nodules in a cohort of dogs with spirocercosis associated caudal esophageal nodules.

\section{Materials and Methods}

All procedures were approved by and conducted in accordance with institutional animal care requirements. Thirty-eight dogs with spirocercosis were prospectively recruited. All dogs had caudal esophageal nodules that were diagnosed by means of endoscopy within 5 days of radiographic and CT examinations. Dogs were classified into neoplastic or non-neoplastic nodule groups based on tissue biopsy, post mortem histology, or response to treatment. All dogs were evaluated over a two-year period (2009-2010).

Thoracic CT was performed with dogs positioned in sternal recumbency. A dual slice helical CT scanner (Siemens Emotion Duo, Erlangen, Germany) was used for all dogs and technique settings included $3 \mathrm{~mm}$ slices, a pitch of 1.95 , and $1.5 \mathrm{~mm}$ thick slice reconstructions. All dogs were under general anesthesia with forced maintenance of an inspiratory phase using positive pressure ventilation techniques. An additional endotracheal tube was placed in the esophagus and inflated with air prior to CT examination in order to optimize visibility of esophageal nodules. Precontrast and three postcontrast scans were acquired for each dog after administration of $2 \mathrm{ml} / \mathrm{kg}$ of iohexol $300 \mathrm{mg} \mathrm{I} / \mathrm{ml}$ at $3 \mathrm{ml} / \mathrm{s}$ using a pressure injector. No saline chaser was used. The precontrast CT was made from C7 to L2, early arterial phase from T3$\mathrm{L} 1$, late arterial phase from $2 \mathrm{~cm}$ cranial to the mass to $2 \mathrm{~cm}$ caudal to the mass and venous 
phase from T3-L1. The length of thoracic area scanned differed to allow for tube cooling and respiration between the scans. All scans were done using bolus tracking with a ROI placed in the aorta at the level of T10 and triggering the postcontrast scans at $200 \mathrm{HU}$. This was monitored and if for some reason, the trigger was not activated (e.g. respiratory motion moving ROI away from aorta) the injection was manually triggered which happened in two dogs. The early arterial scan started $6 \mathrm{~s}$ post trigger, late arterial $35 \mathrm{~s}$ post trigger and venous scan 65 s post trigger.[14] All post contrast scans followed automatically on each other after preprograming all scan delays. Precontrast CT scans were done at $130 \mathrm{kV}$ and postcontrast scans at $110 \mathrm{kV}$, all at tube rotation time of $0.8 \mathrm{~s}$. The effective mAs varied but was generally 2-4 mAs lower for post contrast scans. The pressure injector was set at 200 psi but resultant pressures ranged from 39 to 67 with a mean of $67 \mathrm{psi}$. Set dosage of $2 \mathrm{ml} / \mathrm{kg}$ and injection speed of $3 \mathrm{ml} / \mathrm{s}$ varied minimally on postinjection evaluation.

All CT examinations were reloaded onto the CT computer and interpreted by a single board certified radiologist (RK) who was unaware of clinical or radiographic findings. Images were viewed in bone and mediastinal windows. Window levels, window widths, and magnification were adjusted as needed to optimize visualization of lesions in image reconstructions.

Nodules were examined and surface characteristics described as smooth, slightly irregular or irregular. Additionally, the following were recorded: A subjective percentage was recorded of the nodule/neoplastic mass that was suspected to be abscessation or necrosis as indicated by hypoattenuating areas $(<25 \mathrm{HU})$ without contrast enhancement; and the presence of mineralization. The tracheobronchial lymph nodes were examined for enlargement or abnormal contrast uptake.

The largest possible ROIs were taken of the normal esophageal wall adjacent to the nodule, as well non-neoplastic and neoplastic nodules (excluding the wall)[14] at three different transverse slices and mean HU as well as standard deviations (SD) recorded and averaged for precontrast and the three post contrast phases. The ROIs were placed in the same position on the same slice in each phase. In cases with multiple nodules the largest was selected. Necropurulent areas with no perfusion and with $\mathrm{HU}<25$ as well as areas of mineralization were excluded from the ROI.[14] Heterogeneity of contrast uptake was defined as the average SD per group. Heterogeneity was absent if the SD was $<10$ than the mean HU, mild if the SD was $>10$ and $<20$ the mean HU, moderate 20 to $<30$ and marked $>30$ HU.[26]

Confirmation of a $S$. lupi esophageal nodule or mass was made in all patients by means of esophageal and gastric endoscopies (Olympus GIF video endoscope, type XQ200, Tokyo, Japan) usually immediately post CT. Each dog was placed in left lateral recumbency and a complete gastroesophageal endoscopy was performed. Biopsies were taken of suspected neoplastic nodules.

Statistical tests were selected and performed by one of the co-authors (ED), using commercially available statistical analysis software programs (Excel 97-2003® Microsoft Corporation, Redmont, WA98052, USA and IBM SPSS Statistics 21, USA). The HU measurements and their SD showed normal distribution tested by One-Sample Kolmogorov Smirnov Test and consequently Student's $t$-test was used to determine which group's (neoplastic or non-neoplastic) perfusion phases showed the highest attenuation as well as comparing normal esophageal wall attenuation differences between the two groups. The proportion of mass changes (mineralization, puss formation etc.) was determined for each nodule and compared between groups. Results of comparisons were expressed as mean \pm 
standard deviation. The prevalence of different patterns of the nodule surface (smooth, slightly irregular and irregular) was compared between groups using Chi square test. The significance level for all tests was set at $P<0.05$.

\section{Results}

\section{Dogs}

There were 38 dogs with age and sex distribution given in Table 1 There were five German Shepherd dogs, four Jack Russell terriers, and Boerboels; and two each of Boxers, Staffordshire terriers, Scottish terriers, pit bull terriers, and Labrador retrievers. The remaining 15 dogs were breeds with one dog each or cross breed dogs. There were 14 intact and seven sterilized females, and 13 intact and four sterilized males. There was no significant difference in age or prevalence of gender between groups (Table 1). Twenty-three dogs had non-neoplastic nodules and 15 dogs had neoplastic nodules of which 14 were osteosarcomas with one fibrosarcoma.

\begin{tabular}{|lccc|}
\hline \multicolumn{4}{|c|}{ Table 1. Age and Sex Data of Dogs } \\
Non-neoplastic & Neoplastic group & \\
group $\boldsymbol{n}=\mathbf{2 3}$ & $\boldsymbol{n}=\mathbf{1 5}$ & $\boldsymbol{P}$-value \\
& Mean \pm SD & Mean \pm SD & \\
Median and range & Median and range \\
Age in months & $65.35 \pm 45.36$ & $68.13 \pm 23.97$ & 0.83 \\
& $51(10-160)$ & $64(24-120)$ & \\
Sex & 9 F \& 5 FS & 5 F \& 2 FS & 0.47 \\
& 8 M \& 1MS & 5 M \& 3 MS & \\
F, female; FS, female sterilized; M, male; MS, male sterilized &
\end{tabular}

\section{Mass Characteristics}

All the non-neoplastic nodules were smooth while in the neoplastic group 9/15 were slightly irregular and 6/15 were irregular (Figs. 1 and 2). This difference in prevalence of surface pattern was highly significant $(P<0.001)$. Only the neoplastic group showed mineralization within the nodule with 14/15 (93\%) showing some mineralization. The portion of the mass that was mineralized was $17.87 \% \pm 24.6$ and the difference from the non-neoplastic group was highly significant $(P=0.001)$ (Fig. 2). 

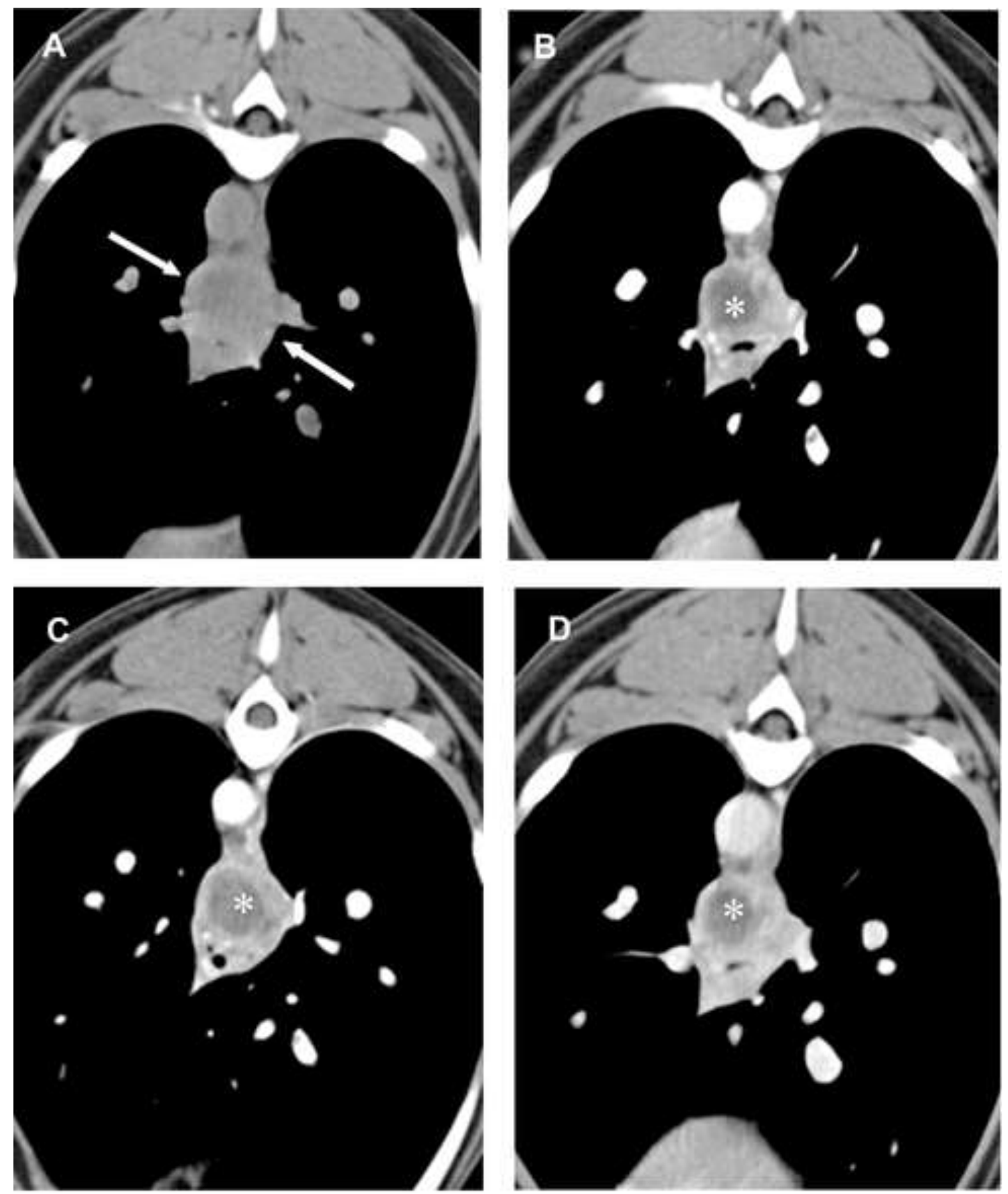

Figure 1. Husky crossbreed spayed female 18 months old with a benign esophageal nodule (dimensions $90 \mathrm{~mm}$ long, $44 \mathrm{~mm}$ wide, and $30 \mathrm{~mm}$ high between white arrows). Transverse CT images all at the same level just caudal to the heart. The left side of the patient is on the left on the images. (A) Precontrast (B) early arterial (C) late arterial and (D) venous phase in a mediastinal window. WW400 WL40. Note the smooth nodule outline and the necropurulent area (white*) within the nodule emphasized by contrast uptake peripherally to it in B-D. The necropurulent material was adjudged to take up $40 \%$ of the overall nodule volume. Average perfusion for this nodule was $37,102,102$, and 97 HUs for the survey and three perfusion phases, respectively. 

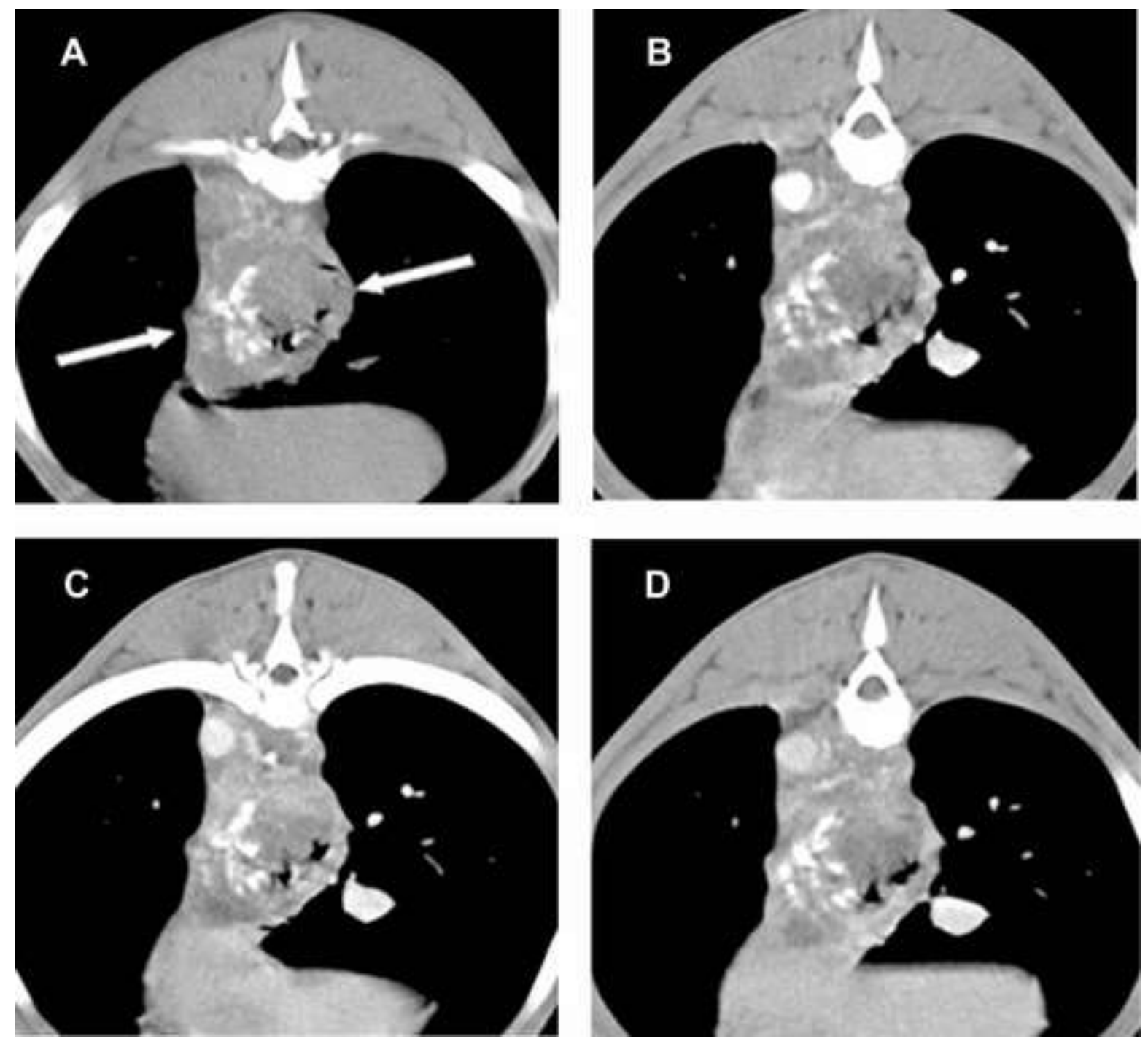

Figure 2. Pitt-bull entire male eight years old with an esophageal osteosarcoma (dimensions $92 \mathrm{~mm}$ long, 52 $\mathrm{mm}$ wide, and $48 \mathrm{~mm}$ high between white arrows). Transverse CT images all at the same level caudal to the heart. The left side of the patient is on the left on the images. (A) Precontrast (B) early arterial (C) late arterial and (D) venous phase in a mediastinal window. WW400 WL40. Note the irregular nodule surface and mineralization on the left of the nodule. The necropurulent material was adjudged to take up $4 \%$ and mineralization $10 \%$ of the overall nodule volume. Average perfusion for this nodule was 42, 64, 63, and 64 HUs for the survey and three perfusion phases, respectively.

The non-neoplastic nodules had necropurulent pockets in 17/23 (73.9\%) cases and showed a significantly $(P=0.02)$ higher proportion of nodule involvement $(18.61 \% \pm 21.28$ of the masses) compared to the neoplastic nodule involvement $(4.27 \% \pm 7.76)$ despite $7 / 15(46.7 \%)$ dogs having necropurulent pockets (Figs. 1 and 2). There was no evidence of regional lymphadenopathy in any of the dogs.

The contrast enhancement of the non-neoplastic nodules was significantly higher when compared to the enhancement of the neoplastic nodules during all three phases (Table 2 and Figs. 1 and 2). Surprisingly the same trend was seen in the enhancement of the normal adjacent esophagus of the non-neoplastic group compared to the neoplastic group and the difference was significant in the survey and early arterial phases (Table 2). The normal esophagus adjacent to the neoplastic nodules consistently had a higher HU compared to the 
neoplastic nodule for all phases whereas the same was only true for the survey and early arterial phases for the non-neoplastic nodules.

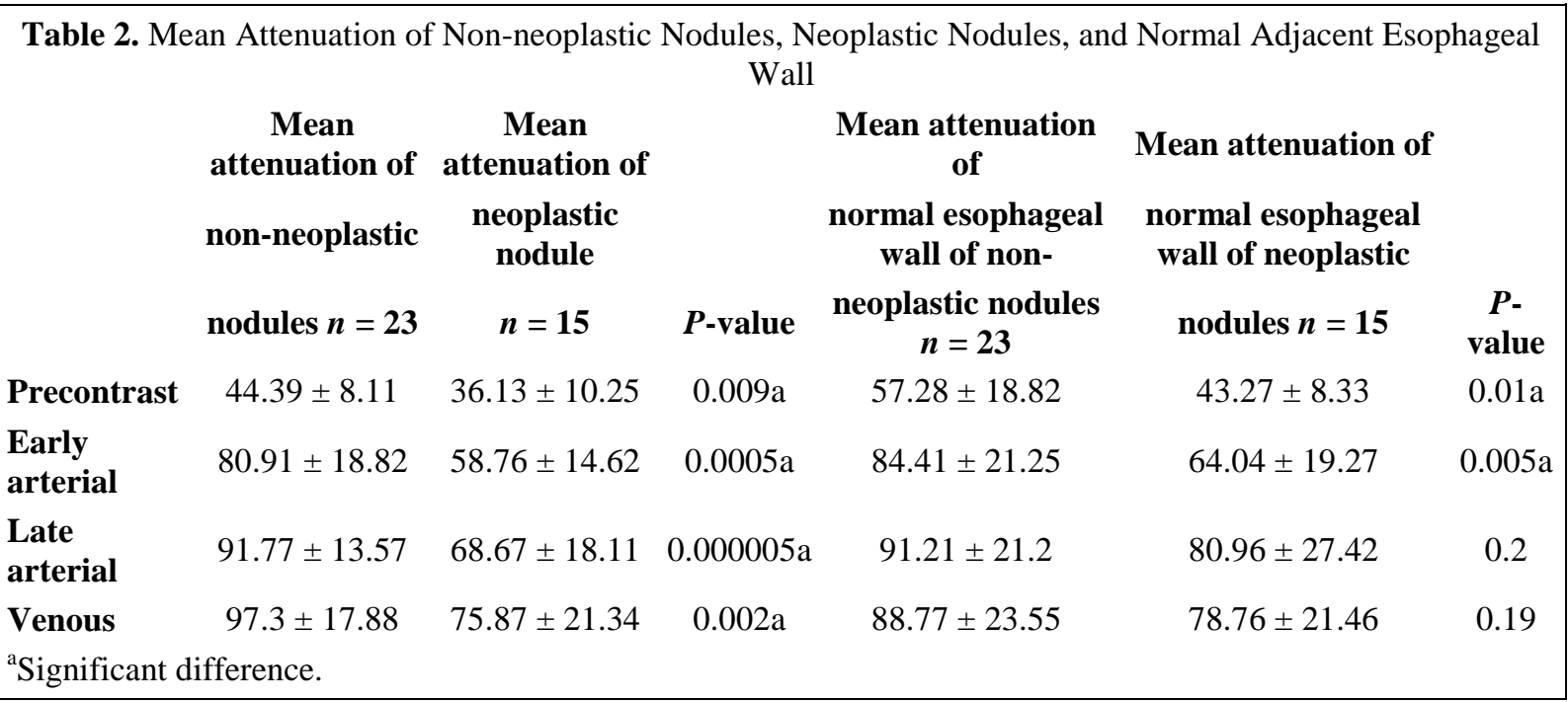

The ratios of contrast enhancement between the normal esophageal wall and the nodules of the non-neoplastic vs. the neoplastic nodules did not differ significantly during all perfusion phases (Table 3). The heterogeneity of non-neoplastic and neoplastic nodules did not differ significantly in any of the postcontrast phases.

Table 3. Mean Ratio of Normal Esophagus vs. Adjacent Non-neoplastic Nodules and Adjacent Neoplastic Nodules

Mean ratio of normal
esophagus vs. nodule

CT phase

Pre contrast

Early arterial

Late arterial

Venous

$$
\begin{gathered}
\boldsymbol{n}=\mathbf{2 3} \\
0.83 \pm 0.21 \\
0.96 \pm 0.89 \\
1.04 \pm 0.21 \\
1.15 \pm 0.28
\end{gathered}
$$

\begin{tabular}{cc}
$\begin{array}{c}\text { Mean attenuation of normal } \\
\text { esophagus vs. neoplastic } \\
\text { nodule } \boldsymbol{n}=\mathbf{1 5}\end{array}$ & $\boldsymbol{P}$-value \\
$0.87 \pm 0.32$ & 0.69 \\
$0.92 \pm 0.76$ & 0.09 \\
$0.93 \pm 0.33$ & 0.23 \\
$1.00 \pm 0.27$ & 0.12 \\
\hline
\end{tabular}

\section{Discussion}

Esophageal CT examination showed significant differences between non-neoplastic and neoplastic spirocercosis associated esophageal nodules. Non-neoplastic nodules were smooth and nonmineralized with a higher proportion of necropurulent filled cavities. In $74 \%$ of nonneoplastic nodules hypoattenuating areas were present with an average of $19 \%$ of the mass surface area involved compared to $47 \%$ of neoplastic nodules that involved an average of $4 \%$ of mass surface area. Neoplastic nodules had a more irregular surface with up to $93 \%$ of cases having mineralized foci taking up an average of $18 \%$ of their cross sectional surface area. Non-neoplastic nodules were significantly more perfused than neoplastic nodules. The normal esophagus was also significantly more perfused than the adjacent neoplastic nodules. These findings were indicative of relative hypoperfusion of $S$. lupi associated esophageal sarcomas.

The CT findings of a smooth surface in nonplastic nodules vs. a more irregular nodule surface in neoplastic nodules corresponds with described endoscopy findings of irregular, 
often cauliflower-like surface which may be discolored with necrotic surface patches, being associated with neoplastic transformation. [2, 8] Whilst routine CT cannot compare to endoscopy to characterize these findings the CT changes are a useful adjunct in helping to make a decision on neoplastic transformation. Mass edge visibility is enhanced by introducing air into the esophagus. However there is a limit as to how much air remains in the esophagus during the procedure as excess amounts are often expelled again. The air readily surrounds the small nodules but with large masses this cannot always be achieved and often only the cranial or caudal borders can be evaluated theoretically making it possible to miss an irregular border. Virtual CT endoscopy may also have a role to play to identify surface characteristics providing that adequate esophageal gas distension is obtained and this modality needs further investigation.

The dilemma in interpreting mass mineralization on radiographs is that trapped mineralized debris can easily be confused with mass mineralization. The cross sectional images obtained with CT makes misdiagnosing debris as mineralized mass less likely but even in the current CT study one could not always be certain of where the mineralization was located.

Endoscopic biopsy is normally done when spirocercosis is diagnosed by means of radiology or fecal analysis but routine CT examinations for other thoracic pathology may detect incidental spirocercosis lesions and using above criteria CT findings can assist to prognosticate as to the malignancy of the nodules

In man, and probably in animals as well, most tumors have the greatest density of microvessels peripherally in the "zone of angiogenesis." [18] As the tumor grows the central portions become relatively hypovascular and eventually undergo necrosis. This is contrary to what was seen in the current study where the benign nodule often had a large area of hypoattenuating tissue due to necrosis and puss formation. This correlated well with the localized areas of necrosis and variable amounts of puss found in cavernous tracts around the worms as described in pathology reports of early cases.[27] This has also been described more recently by our group in histological analyses of non-neoplastic nodules where purulent foci were described adjacent to worm tracts.[3] The hypoattenuating areas seen in our neoplastic cases was minimal and could be attributable to tumor necrosis as a result of the larger sized neoplastic nodules or be remnant foci of necropurulent material from the preneoplastic stage.

In human esophageal squamous cell carcinoma tumor vascularity has been shown to play a role in prognosis as angiogenesis is essential for growth of solid tumors. [19] In nonesophageal tumors increasing tumor vascularity in carcinomas is also associated with increased risk of metastasis but this association is not seen in sarcomas.[19-21] However one canine study on perfusion parameters in tumors[25] concluded that soft tissue sarcomas were less perfused than bone sarcomas and carcinomas which correspond to the fairly hypoperfused neoplastic nodules vs. non-neoplastic nodules in our study. Our perfusion study correlated well with a study comparing MVD using an anti-von Willebrand Factor (Factor VIII) antibody to identify vascular endothelium in normal esophagus and non-neoplastic and neoplastic $S$. lupi associated nodules.[10] The question remains as to how the hypoperfused sarcoma meets the growing need for nutrition?

A limitation of the current study was that a dual slice CT machine was used practically limiting the perfusion assessment to three slices and only measuring $\mathrm{HU}$ as in indication of perfusion rather that whole tumor imaging using more sophisticated equipment and perfusion 
parameters as described above. Additionally the tissues examined did not have comparative microvessel densities determined to corroborate our findings or that biopsy samples were not necessarily from the same nodule that the perfusion study was performed on. In any CTA study timing of arterial, venous, and perfusion stages can never be precisely duplicated due to differences in patient size, heart rate, cardiac output, and blood pressure and this may have influenced our perfusion parameters. Despite these shortcomings we believe that whilst working in a veterinary environment valuable information of nodule perfusion can be obtained despite any possible side effects such as contrast induced nephropathy.

To date this is the first CTA perfusion study evaluating spirocerca associated nodules and their neoplastic transformation and one of very few veterinary articles in this field. Findings from our study indicate that $S$. lupi induced esophageal sarcomas are less perfused than their non-neoplastic nodule counterparts. This relative hypoperfusion together with the less sensitive CT indicators of malignancy (irregular surface, large nodule, mineralization, and minimal necropulent material) all contribute to making a diagnosis of neoplastic transformation on a CT examination. Veterinary oncological studies utilizing a variety of CT perfusion protocols to determine malignancy, tumor type or use in targeted therapies are in their infancy and whilst challenges remain regarding funding and equipment in many institutions, veterinary radiologists need to take their rightful place in expanding and developing CT perfusion studies in animals, including laboratory animal models in early phase drug trials.

\section{REFERENCES}

1. Bailey WS. Spirocerca lupi: a continuing inquiry. J Parasitol 1972;58:3-22.

2. Van der Merwe LL, Kirberger RM, Clift S, Williams M, Keller N, Naidoo V. Spirocerca lupi infection in the dog: a review. Vet J 2008;176:294-309.

3. Dvir E, Clift SJ, Williams MC. Proposed histological progression of the Spirocerca lupiinduced oesophageal lesion in dogs. Vet Parasitol 2010;168:71-77.

4. Dvir E, Kirberger RM, Malleczek D. Radiographic and computed tomographic changes and clinical presentation of spirocercosis in the dog. Vet Radiol Ultrasound 2001;42:119129.

5. Christie J, Schwan EV, Bodenstein LL, Sommerville JEM, van der Merwe LL. The sensitivity of direct faecal examination, direct faecal flotation, modified centrifugal faecal flotation and centrifugal sedimentation/flotation in the diagnosis of canine spirocercosis. $J S A$ Vet Ass 2011;82:71-75.

6. Seibold HR, Bailey WS, Hoerlein BF, Jordan EM, Schwabe CW. Observations on the possible relation of malignant esophageal tumors and Spirocerca lupi lesions in the dog. Am J Vet Res 1955;16:5-14.

7. Kirberger RM, Stander N, Cassel NN, et al. Computed tomographic and radiographic characteristics of aortic lesions in 42 dogs with spirocercosis. Vet Radiol Ultrasound 2013:54;212-222. 
8. Ranen E, Lavy E, Aizenberg I, Perl S, Harrus S. Spirocercosis-associated esophageal sarcomas in dogs. A retrospective study of 17 cases (1997-2003). Vet Parasitol 2004;119:209-221.

9. Dvir E, Kirberger RM, Mukorera V, van der Merwe LL, Clift SJ. Clinical differentiation between dogs with benign and malignant spirocercosis. Vet Parasitol 2008;155:80-88.

10. Dvir E, Clift SJ. Evaluation of selected growth factor expression in canine spirocercosis (Spirocerca lupi)-associated non-neoplastic nodules and sarcomas. Vet Parasitol 2010;174:257-266.

11. Mukorera V, Kirberger RM, Mabeta P, Dvir E. Vascular endothelial growth factor concentrations in dogs with spirocercosis. J Vet Int Med 2013;27:1642-1645.

12. Lavy E, Aroch I, Bark H, et al. Evaluation of doramectin for the treatment of experimental canine spirocercosis. Vet Parasitol 2002;109:65-73.

13. Austin CM, Kok DJ, Crafford D, Schaper R. The efficacy of a topically applied imidacloprid 10\%/moxidectin $2.5 \%$ formulation (Advocate ${ }^{\circ}$, Advantage $\circledR$ Multi,Bayer) against immature and adult Spirocerca lupi worms in experimentally infected dogs. Parasitol Res 2013;112:S91-S108.

14. Umeoka S, Koyama T, Toogashi K, et al. Esophageal cancer: evaluation with triple phase dynamic CT - initial experience. Radiology 2006;239:777-783.

15. Ridgway RL, Suter PF. Clinical and radiographic signs in primary and metastatic esophageal neoplasms of the dog. J Am Vet Med Assoc 1979;174:700-704.

16. Wandera JG. Further observations on canine spirocercosis in Kenya. Vet Rec 2003; 153:718-720.

17. Lindsay NL, Kirberger RM, Williams M. Spirocercosis associated spinal cord chondrosarcoma. Vet Radiol Ultrasound 2010;51:614-616.

18. Miles KA. Tumour angiogenesis and its relation to contrast enhancement on computed tomography: a review. Eur Radiol 1999;30:198-205.

19. Tanigawa N, Matsumura M, Amaya $\mathrm{H}$, et al. Tumor vascularity correlates with the prognosis of patients with esophageal squamous cell carcinoma. Cancer 1997;79:220-225.

20. Tomlinson J, Barsky SH, Nelson S, et al. Different patterns of angiogenesis in sarcomas and carcinomas. Clin Cancer Res 1999;5:3516-3522.

21. West CC, Brown NJ, Mangham DC, Grimer RJ, Reed MWR. Microvessel density does not predict outcome in high grade soft tissue sarcoma. J Cancer Surg 2005;31:1198-1205.

22. Love NE, Fisher P, Hudson L. The computed tomographic enhancement patterns of the normal pituitary gland. Vet Radiol Ultrasound 2000;41:507-510. 
23. Van der Vlugt-Meijer RH, Meij BP, Voorhout G. Dynamic computed tomographic evaluation of the pituitary gland in healthy dogs. Am J Vet Res 2004;65:1518-1524.

24. Van der Vlugt-Meijer RH, Voorhout G, Meij BP. Imaging of the pituitary gland in dogs with pituitary-dependant hyperadrenocorticism. Mol Cell Endocrinol 2002;197:81-87.

25. Nitzi D, Ohlerth S, Mueller-Schwandt F, Angst A, Roos M, Kaser-Hotz B. Dynamic computed tomography to measure tissue perfusion in spontaneous canine tumours. Vet Radiol Ultrasound 2009;50:347-352.

26. Spector DI, Fischetti AJ, Kovak-McClaren JR. Computed tomographic characteristics of intrapelvic masses in dogs. Vet Radiol Ultrasound 2011;52:71-74.

27. Bailey WS. Parasites and cancer: sarcoma in dogs associated with Spirocerca lupi. Ann NY Acad Scie 1963;108:890-923. 